\title{
Hidroclorotiazida y espironolactona reducen la hipertrofia de la pared aórtica en la hipertensión arterial experimental
}

\author{
David Mondaca ${ }^{1,2}$, Patricio Araos ${ }^{1,3}$, Cristián Yañez ${ }^{1,2}$, \\ Ulises Novoa', María Paz Ocaranza ${ }^{\dagger}$, Jorge Jalil ${ }^{\dagger}$
}

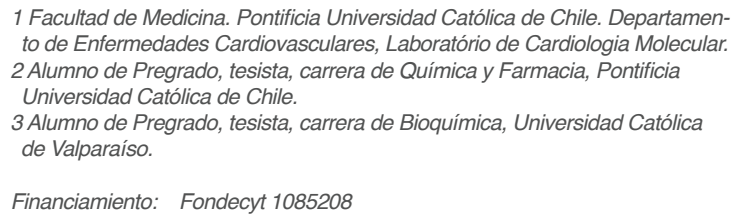

\section{Resumen:}

Introducción: Los diuréticos forman parte del tratamiento antihipertensivo actual con efectividad e impacto clínico demostrados. Sin embargo, los efectos de estos fármacos sobre el remodelado de la pared arterial en la hipertensión arterial (HTA) han sido poco evaluados.

Objetivos: Determinar y comparar el efecto de Hidroclorotiazida (HCTZ) y de Espironolactona (ESP) en la hipertrofia de la pared aórtica en la HTA experimental.

Metodología: Estudio comparativo en 4 grupos experimentales. Se utilizaron ratas Sprague Dawley macho de $150 \pm 10$ grs. unifrectomizadas tratadas con desoxicorticosterona Acetato (DOCA, $100 \mathrm{mg} / \mathrm{Kg} / \mathrm{sem}$ sbc) por 6 semanas. Como controles (Sham) se usaron ratas unifrectomizadas. A partir de la tercera semana con DOCA se administró diuréticos en dos grupos adicionales durante 3 semanas. Uno recibió HCTZ (6 mg/ $\mathrm{kg} /$ día) y otro ESP $(100 \mathrm{mg} / \mathrm{Kg} /$ dia $)$, vía gavage. Al fi- nalizar los tratamientos se determinó la presión arterial sistólica (PAS), masa corporal (MC), peso del corazón (PC) y masa cardiaca relativa (MCR). El grado de hipertrofia de la pared aórtica se determinó midiendo el grosor de la túnica media (GTM), área de la túnica media (ATM), área luminal (AL) y la relación ATM/AL en cortes teñidos con hematoxilina-eosina.

Resultados: En las ratas DOCA no tratadas hubo un aumento significativo de PAS (51\%), MCR (79\%), ATM (44\%), GTM (57\%), y de la razón ATM/AL (43\%) respecto al grupo Sham. Ambos tratamientos (Hctz y Esp) redujeron en forma muy importante y significativamente la PAS, MCR, ATM, GTM y la razón ATM/AL en magnitudes similares y también por cada mm de $\mathrm{Hg}$ de descenso de la PAS logrado.

Conclusión: Además del efecto antihipertensivo, tanto hidroclorotiazida como espironolactona previenen y/o revierten en magnitud similar el desarrollo de hipertrofia de la pared aórtica en este modelo de HTA experimental.

Correspondencia:

Dr Jorge Jalil,

Departamento de Enfermedades

Cardiovasculares, PUC, Marcoleta 367 Piso 8,

jjalil@med.puc.cl 


\section{Diuretics are important and effective antihypertensive agents. However, there are a few studies assessing its role on the arterial wall remodelling in hypertension .}

Aims: To determine and compare the effects of hydrochlorotiazide (Hctz) and spironolactone (Esp) on hypertrophy of the aortic wall in experimental hypertension.

Methods: This was a comparative study with 4 experimental groups. We used male. uninephrectomized Sprague Dawley rats $(150 \pm 10$ grs $)$ treated with desoxycorticosterone acetate (DOCA, $100 \mathrm{mg} / \mathrm{Kg} /$ week sbc) during 6 weeks. As controls uninephrectomized rats (Sham) were used. Starting from the third week with DOCA, two groups recived diuretics by gavage during 3 weeks. One group received $\mathrm{Hctz}(6 \mathrm{mg} / \mathrm{kg} /$ day) and other group received $\mathrm{Sp}(100 \mathrm{mg} / \mathrm{kg} / \mathrm{day})$. At the end of the study, systolic blood pressure (SBP), body weight, heart weight and relative cardiac weight were measured. Hypertrohy in the aortic wall was as-

\section{Introducción}

La hipertensión arterial (HTA), definida como un aumento sostenido de la presión arterial sistólica (PAS) mayor o igual a $140 \mathrm{mmHg}$ y/o mayor o igual a $90 \mathrm{mmHg}$ de presión diastólica (PD) ${ }^{1}$, es una enfermedad multisistémica que afecta aproximadamente al $26 \%$ de la población mundial $^{2}$. En Chile, la prevalencia actual estimada de HTA es $26.9 \%^{3}$.

Durante la larga evolución de esta enfermedad fundamentalmente asintomática se produce daño en órganos blanco, principalmente cardiovascular, cerebrovascular y renal. Posteriormente, aparecen eventos clínicos severos que llevan a discapacidad, disminución de la calidad de vida y elevada mortalidad. Subyacente a estos procesos está el remodelado patológico secundario a la HTA que se observa particularmente arterial, pero también en corazón, riñón y cerebro. Uno de los desafíos actuales en el manejo de la HTA, además de lograr la normotensión, es la prevención/regresión del remodelado cardiovascular patológico caracterizado por cambios celulares y tisulares como hipertrofia, fibrosis e inflamación cardiovascular, así como también disfunción endotelial, alteración de la matriz extracelular y estrés oxidativo ${ }^{4,5,6}$.

Con el tratamiento antihipertensivo actualmente disponible se ha logrado reducir en aproximadamente un $40 \%$ los accidentes cerebrovasculares y en $20 \%$ la incidencia de sessed by measuring the media thickness (MT), media area (MA), lumen area (LA) and by the AM/LA ratio on hematoxyline-eosine stained cross sections.

Results: Compared to the Sham group, in the untreated hypertensive DOCA group, SBP and relative cardiac weight increased significantly (by 51\% and $79 \%$, respectively), MA increased by $44 \%$, as well as MT (57\%) and the AM/LA ratio (43\%). Both treatments (Hctz and $\mathrm{Sp}$ ) reduced importantly and significantly SBP, relative cardiac mass as well as MT, MA and the $\mathrm{AM} / \mathrm{LA}$ ratio at a similar extent and by $\mathrm{mm} \mathrm{Hg}$.

Conclusion: Besides the antihypertensive effect, both hydrochlorotiazide and spironolactone prevent and/or regress aortic wall hypertrophy in this experimental model of hypertension.

eventos coronarios.

Los diuréticos tiazídicos o tiazidas forman parte importante del tratamiento de la HTA, utilizándose ampliamente. Producen natriuresis y disminución de sodio corporal 7,8. Las tiazidas fueron descubiertas en 1957 y desde esa fecha se ha demostrado que disminuyen la presión arterial eficientemente en la HTA 9 . Las tiazidas, como la hidroclorotiazida (Hctz), actúan por inhibición del cotransporte sodio-cloruro en la membrana apical de las porciones iniciales del túbulo distal, donde normalmente se reabsorbe cerca del 5-8\% del sodio filtrado en el glomérulo ${ }^{10}$. En el largo plazo, el efecto antihipertensivo de las tiazidas se debe a disminución de la resistencia vascular sistémica.

Por otro lado, los bloqueadores del receptor de mineralocorticoide (RM) como espironolactona (Esp), también conocidos como antagonistas de aldosterona, actúan en el túbulo contorneado distal y colector, donde el sodio es reabsorbido por los canales EnaC o sensibles a amiloride. En este lugar el sodio es intercambiado por potasio mediante la bomba $\mathrm{Na}+\mathrm{K}+$ ATPasa que depende de la activación de los receptores $\mathrm{RM}^{11}$. Al activarse los receptores RM por su unión con aldosterona, se produce regulación transcripcional de genes involucrados en la homeostasis del agua y sodio, que van a interactuar con los canales EnaC (u otros) lo que produce reabsorción de sodio (y agua) con lo que aumenta la presión arterial ${ }^{12}$. Lo anterior es referido como efectos genómicos de aldosterona. Al- 
dosterona posee además efectos no genómicos o directos (fibrosis, inflamación y remodelado cardíaco y vascular) que se acentúan en presencia de alta ingesta de sodio. La unión de Esp al RM lo inhibe competitivamente, con lo que se produce natriuresis (con ahorro de potasio) y disminución de la presión arterial.

Existen pocos estudios experimentales que evalúen en conjunto el efecto antihipertensivo y antiremodelado vascular de estos diuréticos, que puedan explicar el impacto clínico de estos fármacos de amplio uso en el tratamiento de la HTA. Planteamos que ambos diuréticos pueden tener un rol adicional como fármacos antiremodelado vascular, además de su efecto antihipertensivo. En consecuencia, el objetivo fue evaluar en un modelo experimental de HTA la posible participación de Hctz y de Esp en el remodelado de la pared aórtica. Utilizamos el modelo de HTA experimental en ratas DOCA-sal con unifrectomia lateral, caracterizado por presentar expansión en el volumen extracelular ${ }^{13}$, hipertrofia cardiovascular y esclerosis renal, junto con baja actividad del sistema renina-angiotensina ${ }^{14}$.

\section{Materiales y métodos}

El presente trabajo se realizó de acuerdo a la "Guía para el cuidado y uso de animales de laboratorio" publicado por el "National Health Institute" (NIH No 85-23, 1985) y fue aprobada por la Comisión de Investigación de la Facultad de Medicina de la Pontificia Universidad Católica de Chile.

Se utilizaron ratas Sprague Dawley macho de $150 \pm 10$ grs. A las cuales se les indujo HTA, utilizando un modelo por sobrecarga de volumen mediante la administración del mineralocortioide deoxicorticosterona (acetato, DOCA) y sal. Bajo anestesia intraperitonial con ketamina (35 mg/ $\mathrm{kg}$ de peso) y xilazina ( $7 \mathrm{mg} / \mathrm{kg}$ de peso) se realizó una nefrectomía izquierda. 48 horas después de la cirugía se administró DOCA (100 mg/kg/semana, Steraloids. Inc., Newport, RI, USA) vía subcutánea durante 6 semanas. La sal se administró en el agua de beber $(1 \% \mathrm{NaCl}$ y $0,4 \%$ de $\mathrm{KCl})$.

A partir de la tercera semana de administración de DOCA, las ratas hipertensas fueron divididas en 3 grupos de manera aleatoria: 1) ratas a las que se administró suero fisiológico vía gavage y DOCA subcutáneo semanal por 3 semanas (completando 6 semanas de DOCA, grupo DOCA; 2) ratas a las que se administró el antagonista del receptor de mineralocorticoide, espironolactona (100 $\mathrm{mg} / \mathrm{kg} /$ día, Laboratorios Recalcine, Santiago de Chile) vía gavage durante 3 semanas además de DOCA (grupo DOCA-Espironolactona) y 3) ratas a las que se administró hidroclorotiazida (6 mg/kg/día, Farmacias Reccius, Santiago de Chile) vía gavage durante 3 semanas además de DOCA (grupo DOCA-Hidroclorotiazida). Como grupo control se utilizaron ratas sometidas a nefrectomía izquierda, que recibieron solamente suero fisiológico vía gavage por 6 semanas (grupo Sham). La presión arterial sistólica se midió semanalmente en cada rata por pletismografía en la cola de los animales previa anestesia suave con éter etílico.

Una vez finalizadas las seis semanas de tratamiento total, los animales fueron sacrificados, bajo anestesiD - sd mina $\mathrm{H} \square \mathrm{ng}$ mg de Neso Hila ina $\mathrm{H}$ mg g de 
Cada grupo experimental estuvo constituido por 9 - 12 animales. Para las comparaciones se usó análisis estadístico con ANOVA de un factor seguido por prueba de tStudent-Newman-Keuls. El análisis estadístico se realizó usando el programa GraphPad Prism 5. Se consideró un valor de $\mathrm{p}<0,05$ como estadísticamente significativo.

\section{Resultados}

(Tabla 1).

A las 6 semanas de tratamiento total, las ratas que recibieron exclusivamente DOCA aumentaron la PAS en $60 \mathrm{~mm}$ $\mathrm{Hg}$ en promedio $(\mathrm{p}<0,05)$ respecto a las ratas Sham. Tanto espironolactona (Esp) como hidroclorotiazida (Hctz), administradas durante 3 semanas redujeron la PAS en forma similar y levemente superiores a las observadas en las ratas Sham (diferencia de PAS en promedio $=11 \mathrm{~mm} \mathrm{Hg}$ ).

\begin{tabular}{|c|c|c|c|c|}
\hline & Sham & DOCA & $\begin{array}{c}\text { DOCA- } \\
\text { Esp }\end{array}$ & $\begin{array}{c}\text { DOCA- } \\
\text { Hctz }\end{array}$ \\
\hline PAS (mmHg) & $117 \pm 3$ & $177 \pm 4^{*}$ & $128 \pm 1^{\star} \#$ & $128 \pm 1$ \#\# \\
\hline $\mathrm{MC}(\mathrm{g})$ & $347 \pm 16$ & $183 \pm 8^{*}$ & $322 \pm 9 \#$ & $296 \pm 16^{\star}$ \\
\hline $\mathrm{PC}(\mathrm{g})$ & $1,15 \pm 0,07$ & $1,03 \pm 0,02$ & $1,14 \pm 0,03$ & $1,15 \pm 0,03$ \\
\hline MCR & $0,33 \pm 0,003$ & $0,59 \pm 0,01^{*}$ & $0,4 \pm 0,01 \#$ & $0,4 \pm 0,02 \#$ \\
\hline
\end{tabular}

Valores expresados como promedio $\pm E S ; n=9$ - 12/grupo. Abreviaciones: PAS, presión arterial sistólica; $P C$, peso del corazón; MC, masa corporal; MCR: masa cardíaca relativa. Símbolos: * = $p<0,05$ vs Sham; $\#=p<0,05$ vs DOCA (después de ANOVA significativo).

Como es habitual en este modelo experimental, en las ratas hipertensas por DOCA se observó una reducción del peso corporal de $47 \%$ ( $p<0,05)$ respecto a las ratas Sham, lo que fue significativamente revertido con Esp y con Hctz.

En las ratas hipertensas sin tratamiento, se observó además hipertrofia cardíaca con aumento del $79 \%(\mathrm{p}<0,05)$ de la MCR con disminución importante y similar al utilizar tanto Esp como Hctz.

\section{Hipertrofia de la pared aórtica}

(Tabla 2 y Figuras 1 - 4).

En las ratas hipertensas sin tratamiento se observó un aumento significativo del GTM, del ATM y de la razón
ATM/AL en $57 \%, 44 \%$ y $43 \%$, respectivamente, respecto a sus controles Sham. En ambos grupos DOCA tratados con Esp o con Hctz se observó una disminución significativa del ATM y del GTM. La razón ATM/AL se normalizó completamente con ambos tratamientos (Figura 3). Los efectos observados de ambos diuréticos sobre los parámetros de hipertrofia de la pared aórtica fueron mayores al indexar estos parámetros por el peso corporal, con normalización de casi todos ellos (Figura 4).

Por cada mm de $\mathrm{Hg}$ de descenso de la PAS logrado con Hctz o con Esp la reducción de todos los parámetros de hipertrofia de la pared aórtica fue similar (Tabla 3).

\begin{tabular}{|c|c|c|c|c|}
\hline & Sham & DOCA & $\begin{array}{l}\text { DOCA- } \\
\text { Esp }\end{array}$ & $\begin{array}{l}\text { DOCA- } \\
\text { Hctz }\end{array}$ \\
\hline $\mathrm{GTM} \mu \mathrm{m}$ & $96,0 \pm 1,5$ & $151,2 \pm 1,8^{\star}$ & $117,7 \pm 2,1^{*} \#$ & $116,6 \pm 3,9^{*} \#$ \\
\hline ATM mm2 & $0,59 \pm 0,01$ & $0,85 \pm 0,03$ * & $0,67 \pm 0,02 \#$ & $0,62 \pm 0,02 \#$ \\
\hline $\mathrm{AL} \mathrm{mm} 2$ & $1,86 \pm 0,06$ & $1,57 \pm 0,08^{\star}$ & $1,90 \pm 0,05 \#$ & $1,74 \pm 0,07$ \\
\hline ATM/AL & $0,37 \pm 0,03$ & $0,53 \pm 0,02^{*}$ & $0,35 \pm 0,003 \#$ & $0,38 \pm 0,02 \#$ \\
\hline
\end{tabular}

Valores expresados como promedio $\pm E S, n=9-12 /$ grupo. Abreviaciones: GTM, Grosor de la túnica media; $A L$, área luminal; $A T$, área total; $A T M$, área de la túnica media $(A T-A L) ; A T M / A L$, razón ATM/AL. Símbolos: $*=p<0,05$ vs Sham; $\#=p<0,05$ vs DOCA (después de ANOVA significativo).

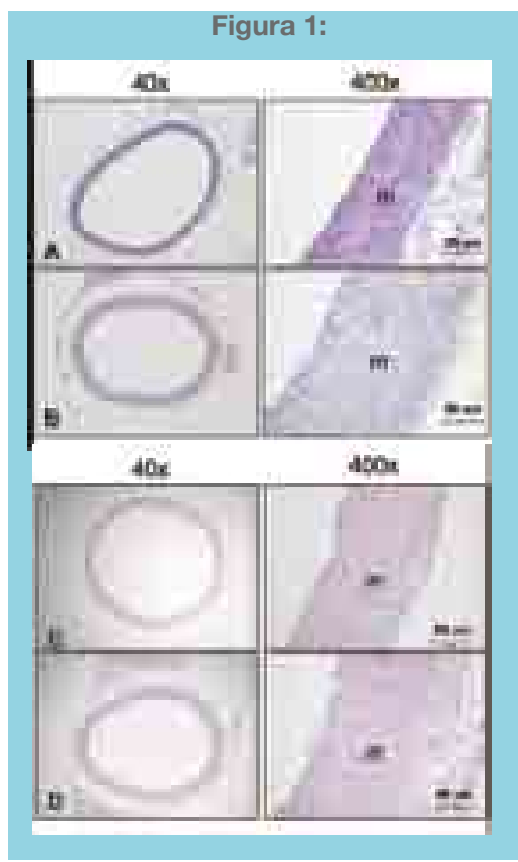

Cortes transversales de aorta teñidos con hematoxilina-eosina representativos de cada grupo experimental. Se observa hipertrofia de la pared aórtica, específicamente de la túnica media $(m)$ en una rata hipertensa DOCA $(B)$ en relación una rata del grupo control Sham (A). Se observa además disminución de la hipertrofia de la pared aórtica, cuando se administra Esp (C) o Hctz (D). Aumento de $40 x$ y $400 x$ por corte. 


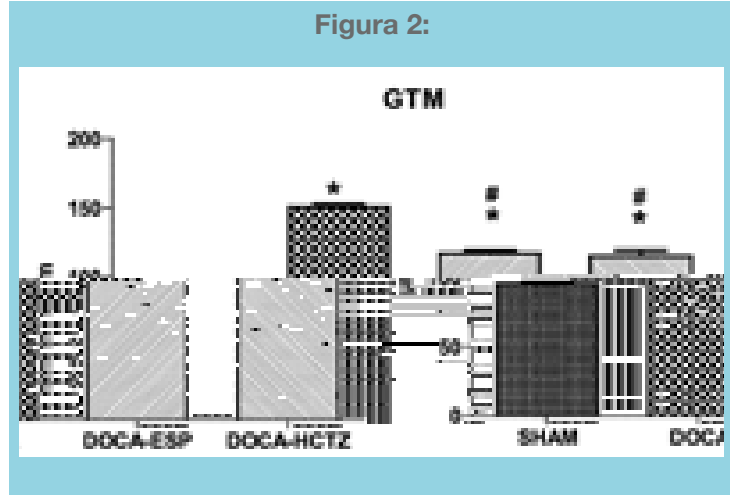

Grosor de la túnica media (GTM) en los 4 grupos experimentales. Valores como promedio $+E S(n=9-12 /$ grupo $)$. Abreviaciones Esp = espironolactona $; \mathrm{Hctz}=$ hidroclorotiazida. Símbolos: $*=p<0,05$ $v / s$ Sham , \# = p < 0,05 v/s DOCA (después de ANOVA significativo).

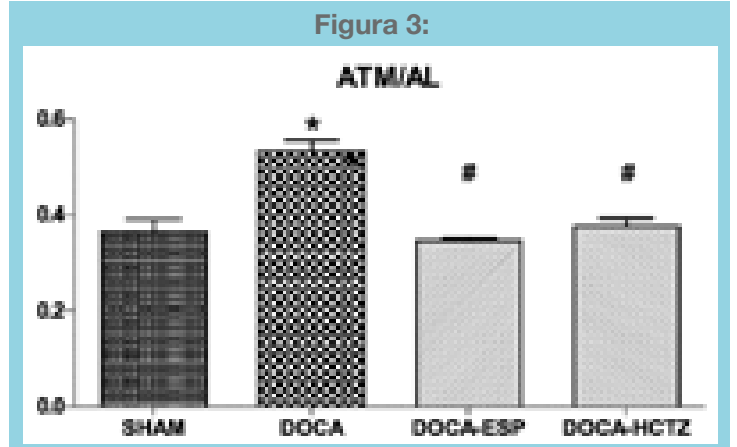

Area de la túnica media en relación al área del lumen (ATM/AL) en los 4 grupos experimentales. Valores como promedio $+E S(n=9$ - 12/ grupo). Abreviaciones Esp = espironolactona; Hctz $=$ hidroclorotiazida. Símbolos: $*=p<0,05 v / s$ Sham, $\#=p<0,05 v / s$ DOCA (después de ANOVA significativo).

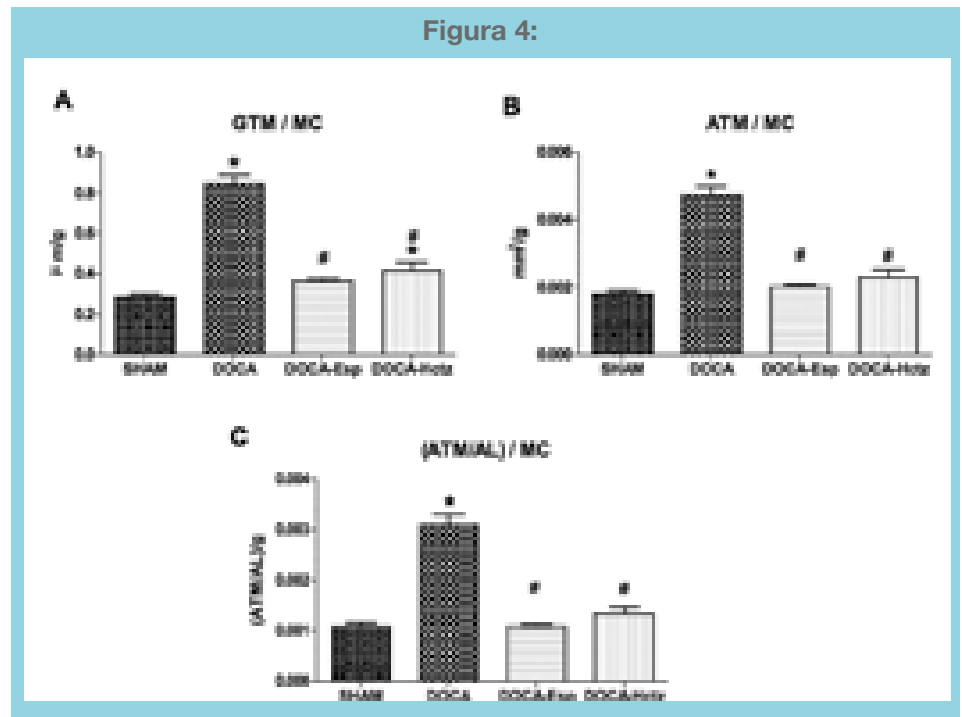

Remodelamiento arterial en relación al peso o masa corporal (MC). Se observa un aumento significativo del grosor de la túnica media (A, ATM/MC), del área de la túnica media $(B, A T M / M C)$ y del área de la túnica media en relación al área del lumen $(C$, [ATM/AL]/MC ) en la ratas hipertensas DOCA y normalización de casi todos estos parámetros con espironolactona (Esp) o con hidroclorotiazida (Hctz) . Valores como promedio + ES $(n=9-12 /$ grupo $)$. Símbolos: $*=p<0,05 \mathrm{v} / \mathrm{s}$ Sham $, \#=p<0,05 \mathrm{v} / \mathrm{s}$ DOCA (después de ANOVA significativo).

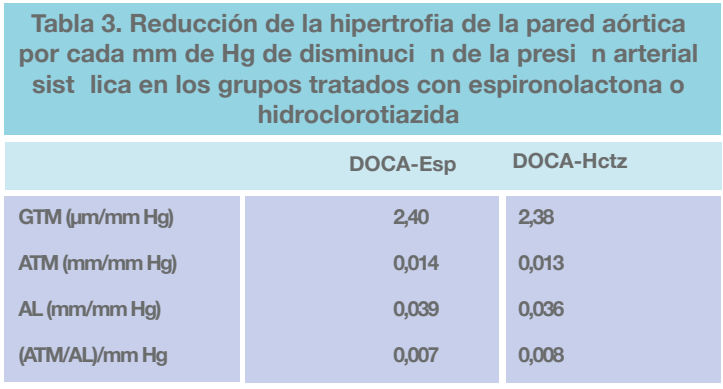

Abreviaciones: GTM, Grosor de la túnica media; AL, área luminal; $A T$, área total; ATM, área de la túnica media $(A T-A L) ; A T M / A L$, razón $A T M / A L$

\section{Discusión}

Nuestros resultados demuestran que en este modelo experimental de HTA por expansión de volumen tanto Hctz como Esp además de disminuir la presión arterial son capaces de reducir en forma muy importante la hipertrofia de la pared aórtica.

El grado de hipertrofia de la pared aórtica desarrollado tras la inducción de HTA estuvo evidenciado por aumento significativo del grosor y del área parietal en el grupo hipertenso DOCA. Además de la importante hipertrofia 
de la pared aórtica que hemos observado en las ratas hipertensas DOCA, en este modelo experimental también se ha observado en la pared aórtica apoptosis, fibrosis, inflamación y mayor expresión de distintas subunidades de NADPH oxidasa 15,16,17,18.

El aumento del ATM y del GTM podría estar relacionado con la pérdida de elasticidad y un aumento en la rigidez de la aorta, aumentando la tensión en la pared vascular y manteniendo la hipertensión arterial. Un aumento de la razón ATM/AL podría provocar aumento en la resistencia al flujo y disminución de las propiedades elásticas de la pared vascular, elevando la tensión o estrés mecánico y la presión intraluminal ${ }^{15,20}$.

Los resultados obtenidos muestran prevención y/o reversión de los parámetros de hipertrofia de la pared aórtica con ambos diuréticos. El GTM, ATM y la razón ATM/ AL disminuyeron significativa y similarmente en las ratas DOCA tratadas con Hctz o con Esp (con idéntico efecto entre ambos fármacos por $\mathrm{mm}$ de $\mathrm{Hg}$ de PAS reducido y también en relación al peso corporal), respecto a aquellas ratas hipertensas que no recibieron tratamiento. Es muy interesante el efecto similar con ambos fármacos que poseen mecanismos de acción muy diferentes, pero logran reducciones similares de la PAS. Lo anterior refuerza la gran importancia de la reducción de la PA como mecanismo protector de remodelado y de daño cardiovascular en la HTA.

Se ha observado que Hctz disminuye la vasoconstricción inducida por Angiotensina II ${ }^{21}$ y que reduce el estrés oxidativo por disminución en la actividad de NADPH Oxidasa y los niveles de ROS, impactando positivamente a nivel estructural y funcional en la pared vascular ${ }^{22}$. De igual manera, Esp disminuye la fibrosis, inflamación y estrés oxidativo cardiovascular mediante un mecanismo similar 23,24. Estos procesos son adicionales a lo que se determinó en el presente estudio (hipertrofia de pared arterial en relación a reducción de PAS) y sugieren posibles mecanismos moleculares adicionales por los cuales Hctz y Esp podrían reducir la hipertrofia de la pared aórtica.

Dentro de las limitaciones del estudio, hubiera sido interesante haber obtenido un correlato funcional de los presentes hallazgos. Por otra parte, la evaluación de paralela de genes y proteínas que favorecen el remodelado vascular patológico podría haber aportado una mayor comprensión de los mecanismos involucrados en los efectos observados con ambos diuréticos, lo que no menoscaba las implicancias de las presentes observaciones. Es muy relevante el hecho de que la magnitud de los efectos antihipetróficos de la pared aórtica con ambos diuréticos sea similar entre ambos a iguales descensos de PAS y también similar a lo que hemos observado recientemente con antihipertensivos de última generación como es el antagonista del receptor de angiotensina candesartán (Mondaca et al, resumen presentado en Congreso Sochicar 2010). Lo anterior parece ir en la línea de que al menos en relación con hipertrofia de la pared arterial en la HTA, el impacto estaría dado principalmente por la disminución de la PAS. No hemos evaluado en este trabajo el impacto sobre arterias más pequeñas o de resistencia, sin embargo, en la pared aórtica de la rata hay un gran número de células musculares lisas que dan cuenta del remodelado hipertrófico (Fig 1). Por último, no es posible extrapolar necesariamente estos resultados a otros modelos de HTA como por ejemplo HTA dependiente de angiotensina II (como en el modelo Goldblatt), en que los efectos de estos fármacos podrían estar atenuados 24

En conclusión, además del efecto antihipertensivo, tanto hidroclorotiazida como espironolactona previenen $\mathrm{y} / \mathrm{o}$ revierten en magnitud similar el desarrollo de hipertrofia de la pared aórtica en este modelo de HTA experimental. Es posible que estos efectos antiremodelado se mantengan en el largo plazo y expliquen una proporción significativa del beneficio clínico de estos fármacos en el tratamiento de la HTA.

\section{Referencias:}

1. KATOVICH MJ, GROBE JL, HUENTELMAN M, RAIZADA MK. Angiotensin-converting enzyme 2 as a novel target for gene therapy for hypertension. Exp. Physiol 2005; 90: 299-305.

2. KEARNEY PM, WHELTON M, REYNOLDS K, MUNTNER P, WHELTON PK, HE J. Global burden of hypertension: analysis of worldwide data. Lancet 2005; 365: 217 223
3. ENS, Encuesta Nacional de Salud 2009-2010, MINSAL. Disponible en: http://epi.minsal.cl/epi/html/secciones/encuestas.htm

4. JACOB MP, BADIER-COMMANDER C, FONTAINE V, BENAZZOUNG Y, FELDMAN L, MICHEL JB. Extracellular matrix remodeling in the vascular wall. Pathol biol 2001; 49: 326-332.

5. GRIENDLING KK, FITZGERALD GA. Oxidative Stress and Cardiovascular Injury: part II: Animal and Human Studies. Circulation 2003; 108: 2034-2040. 
6. VAN DEN BORNE SWM, DIEZ J, BLANKESTEIJN WM, VERJANS J, HOFSTRA L, NARULA J. Myocardial remodeling after infarction: the role of myofibroblasts. Nat Rev Cardiol 2010; 7: 30-37.

7. KRAKOFF LR. Diuretics for Hypertension. Circulation 2005; 112: e127-e129.

8. SHAH SU, ANJUM S, LITTLER WA. Use of diuretics in cardiovascular disease: (2) hypertension. Postgrad Med J 2004; 80: 271-276.

9. SALVETTI A, GUIADONI L. Thiazide Diuretics in the Treatment of Hypertension: An Update. J Am Soc Nephrol 2006; 17: S25-S29.

10. ROSE BD. Diuretic. Kidney Int 1991; 39: 336-352.

11. SAIEH C, ZEHNDER C. DURÁN P, Diuréticos, Hipertensión Arterial, Santiago, Mediterráneo Ltda, 2008: 515-518.

12. TAKEDA Y, YONEDA T, DEMURA M, USUKURA I, MABUCHI H. Calcineurin inhibition attenuates mineralocortiocid-induced cardiac hypertrophy. Circulation 2002; 105: 677-679.

13. LERMAN LO, CHADE AR, SICA V, NAPOLI C. Animal models of hypertnsion: An overview. J Lab Clin Med 2005; 146: 160-173.

14. PINTO YM, PAUL M, GANTEN D. Lessons from rat models of hypertension: from Goldblatt to genetic engineering. Cardiovasc Res 1998; 39: 77-88.

15. INTENGAN HD, SCHIFFRIN EL. Structure and Mechanical Properties of Resistance Arteries in Hypertension: Role of Adhesion Molecules and Extracellular Matrix Determinants. Hypertension 2000; 36: 312-318.

16. INTENGAN HD, SCHIFFRIN EL. Vascular Remodeling in Hypertension: Role of Apoptosis, Inflammation, and Fibrosis. Hypertension 2001; 38: 581-587.
17. JALIL J, NOVOA U, MORA I, OCARANZA MP. La sobreexpresión génica vascular de NADPH oxidasa en la hipertension arterial experimental se reduce solamente con inhibición directa de Rho kinasa. Rev Chil Cardiol 2010; 29: 233-241.

18. TOUYZ RM. Molecular and celular mechanisms regulating vascular function and structure. Implications in the pathogenesis of hypertension. Can J Cardiol 2000; 16: 1137-1146.

19. BRIET M, SCHIFFRIN EL. Aldosterone: effects on the kidney and cardiovascular system. Nat Rev Nephrol 2010; 6:261-273,

20. MAYET J, HUGHES A. Cardiac and vascular pathophysiology in hypertension. Heart 2003; 89:1104-1109.

21. ZHU Z, ZHU S, LIU D, CAO T, WANG L, TEPEL M. Thiazide-Like Diuretics Attenuate Agonist-Induced Vasoconstriction by Calcium Desensitiazation Linked to Rho Kinase. Hypertension 2005; 45: 233-239.

22. CERON CS, CASTRO MM, RIZZI E, MONTENEDRO MF, FONTANA V, SALGADO MCO, et al. Spironolactone and hydrochlorothiazide exert antioxidant effects and reduce vascular matrix metalloproteinase- 2 activity and expression in a model of renovascular hypertension. $\mathrm{Br} \mathrm{J}$ Pharmacol 2010; 160: 77-87.

23. VIRDIS A, NEVES M, AMIRI F, VIEL E, TOUYZ R, SCHIFFRIN E. Spironolactone Improves Angiotensin-Induced Vascular Changes and Oxidative Stress. Hypertension 2002; 40: 504-510.

24. ROCHA R, STIER CT, KIFOR I JR, OCHOA-MAYA MR, RENNKE HG, WILLIAMS GH, et al. Aldosterone: a mediator of myocardial necrosis and renal arteriopathy. Endocrinology 2000; 141: 3871-3878. 